\title{
Fetal Membrane Organ-On-Chip: An Innovative Approach to Study Cellular Interactions
}

\author{
Lauren Richardson, BS ${ }^{1,2}$, Juan Gnecco, $\mathrm{PhD}^{3,4}$, Tianbing Ding, $\mathrm{PhD}^{3}$, \\ Kevin Osteen, PhD $^{3,4}$, Lisa M. Rogers, BS $^{5}$, \\ David M. Aronoff, $\mathrm{MD}^{3,4,5}{ }^{\circ}$, and Ramkumar Menon, $\mathrm{PhD}^{1}$
}

\begin{abstract}
Objective: Fetal membranes, a vital component that helps maintain pregnancy and contribute to parturition signaling, are often studied in segments due to its structural complexity. Transwells are traditionally used to study cell interactions; however, their usefulness is limited. To overcome these difficulties, a fetal membrane-organ-on-chip (FM-OO-C) was created to study interactive properties of amnion epithelial cells (AECs) and decidual cells compared to transwell systems. Methods: Primary AECs and decidual cells from term, nonlaboring fetal membranes were cultured in a 2-chamber (AEC/decidual cell) FM-OO-C device and sandwiched between a semipermeable membrane. Cells were treated with cigarette smoke extract (CSE) or dioxin, and membrane permeability and cellular senescence were measured after 48 hours. The same experiments were conducted in transwells for comparisons. Results: Compared to transwell cultures, FM-OO-C model produced better membrane permeability readings regardless of the side of treatment or time point. Membrane permeabilization was higher in AECs directly treated with CSE ( 1.6 fold) compared to similar treatment on the decidual side (I.2 fold). In FM-OO-C, treatments forced changes between cellular layers. This was evident when CSE and dioxin-induced senescence on one side of the chamber produced similar changes on the opposite side. This effect was minimal in the transwell system. Conclusion: The controlled environment of an FM-OO-C allows for improved signal propagation between cells by minimizing noise and highlighting the small changes between treatments that cannot be seen in conventional transwell devices. Fetal membrane-organ-on-chip provides a better interaction between cell types that can be used to study fetal-maternal signaling during pregnancy in future studies.
\end{abstract}

\section{Keywords}

amnion epithelial cells, decidual cells, fetal membrane organ-on-chip

\section{Introduction}

Human parturition at term is associated with multiple fetal and maternal-derived signals, which coordinate to deliver the fetus. Signals from senescent fetal membranes at term, specifically sterile inflammatory signals, are one of the contributors and initiators of human parturition. ${ }^{1-6}$ Inflammatory signals from fetal tissues are propagated to the maternal tissues, causing immune cell activation and increase decidual inflammation transitioning them to prolabor status. ${ }^{6}$ Our limited understanding of fetal-maternal communications in normal (and diseased) pregnancies is primarily founded upon studies using either intact membrane tissues obtained after deliveries or dispersed cells cultured in isolation. Each of these approaches has significant limitations. ${ }^{7}$ Whole tissue explants have limited viability ex vivo and great subject-to-subject variability, while traditional cell culture systems are plagued by dilution effects reflecting the large volumes of culture media overlying the cells. ${ }^{7}$

To address the issue of studying complex multicellular structures and signaling cascades of tissue and whole organ systems, there has been an evolution away from simple 2-D single cell cultures to coculture (transwell dishes) and

\footnotetext{
' Division of Maternal-Fetal Medicine \& Perinatal Research, Department of Obstetrics \& Gynecology, The University of Texas Medical Branch at Galveston, Galveston, TX, USA

${ }^{2}$ Department of Neuroscience, Cell Biology \& Anatomy, The University of Texas Medical Branch at Galveston, Galveston, TX, USA

${ }^{3}$ Department of Obstetrics and Gynecology, Women's Reproductive Health Research Center, Nashville, TN, USA

${ }^{4}$ Department of Pathology, Immunology, and Microbiology, Vanderbilt University, Nashville TN, USA

${ }^{5}$ Division of Infectious Diseases, Department of Medicine, Vanderbilt University Medical Center, Nashville, TN, USA
}

\section{Corresponding Author:}

Ramkumar Menon, Division of Maternal-Fetal Medicine and Perinatal Research, Department of Obstetrics and Gynecology, The University of Texas Medical Branch, 30I University Blvd, Galveston, TX 77555, USA.

Email: ram.menon@utmb.edu 
cutting-edge 3-D culture (spheroids, organoids) systems to replicate cell-cell and cell-matrix interactions and paracrine communications. ${ }^{8}$ Transwell systems are traditionally used to study cell-cell interactions; however, the usefulness of these static systems is limited, cell migrations are limited and often do not provide reproducible results. ${ }^{9}$ Next generation cell coculture models, termed organ-on-chip (OOC) systems, have revolutionized research and medical fields with the development of the brain, ${ }^{10}$ blood-brain-barrier, ${ }^{11}$ lungs, ${ }^{12,13}$ heart, ${ }^{14}$ and liver ${ }^{15}$-on-chip devices that are currently used to test drug pharmacokinetics and pharmacodynamics, biological processes, and disease phenotypes. Additionally, compared to transwell cultures, the 2-chamber OOC model uses fewer cells and allows for better interaction between cellular layers while providing a sensitive measurement of membrane permeability, cytokine propagation, and signaling pathways.

Recent development of endometrium-on-chip and placentaon-chip has provided insights into dynamic obstetrics complications. ${ }^{16,17}$ These advanced 3-D cell culture systems allow cells to interact with other cells, matrix (eg, collagen), and blood interfaces while maintaining their viability and function. The microfluidic configuration is ideal for testing the effects of drugs, infectious reagents, and endocrine signals on individual cell compartments and their ability to interact and or affect neighboring cells. ${ }^{7}$ Though these models have been developed, additional in utero components such as the umbilical cord, fetal membranes, cervix, and vagina are still lacking complete OOC models.

Fetal membranes, in particular, present an attractive tissue for OOC modeling, since there is a fairly well-defined cellular structure and paracrine signaling appears to be central to its dynamic function throughout normal and diseased gestation. ${ }^{16,18-20}$ It is also a fascinating part of the fetal-maternal interface, containing cells of both fetal and maternal origin cellular layers. A simple single-chamber model of the decidua, the outermost, maternally derived cell layer of the fetal membranes, was recently developed by our group to study paracrine signaling between macrophages and decidual cells in the setting of an inflammatory insult (eg, infection). ${ }^{20}$ However, that model did not incorporate fetally derived cells. Thus, in the present study, we sought, for the first time, to bring together fetal amnion epithelial cells (AECs) and maternal decidual stromal cells to assess paracrine signaling in a microscaled environment. Specifically, we investigated the propagation of fetal signals and their ability to induce changes in maternal cells by creating a 2-chamber fetal membrane organon-chip (FM-OO-C).

The primary objective of this descriptive study was to develop FM-OO-C device and document its potential usefulness by comparing the interactive properties between primary AEC (fetal) and decidual stromal cells (maternal) cocultured in 2-chamber FM$\mathrm{OO}-\mathrm{C}$ device versus traditional transwell plates. Given their relevance to membrane biology,,$^{2,4,21,22}$ we employed models of oxidative stress (OS) and cellular senescence to assess cell-cell interactions in this environment. Using this model system, we determined the effect of 2 environmental toxicants (cigarette smoke and dioxin ${ }^{23}$ ) on AEC and decidual cells separately and tested the impact of exposure effect of one cell type over the other. Cigarette smoke extract (CSE) was chosen as we have shown its ability to induce OS, senescence, and sterile inflammation in AECs and decidual cells. ${ }^{24}$ In addition, OS and associated changes induced by CSE mimic such changes observed in fetal-maternal interface cells from term parturition. The present studies illustrate that the FM-OO-C potentially provides a better window through which interactions between cell types that can be observed in fetal-maternal signaling compared to currently employed transwell systems. The FM-OO-C device controls fluid flow rate and cell viability while allowing real-time observation of the cultured cells and the capacity to analyze complex biochemical and physiological responses.

\section{Methods}

\section{Institutional Review Board Approval}

This study protocol was approved by the institutional review board at The University of Texas Medical Branch (UTMB) at Galveston, Texas, as an exempt protocol for using discarded placenta after standard term cesarean deliveries (UTMB Project 69693). At Vanderbilt University, human gestational membranes were excised from placental tissues from women who delivered healthy, full-term infants by cesarean delivery without labor. Deidentified tissue samples were provided by the Cooperative Human Tissue Network, which is funded by the National Cancer Institute. All tissues were collected in accordance with Vanderbilt University Institutional Review Board (exempt protocol approval \#131607) and Declaration of Helsinki. No subject recruitment or consent was required for this study.

\section{Microfluidic FM-OO-C Design}

Polydimethylsiloxane layer design and fabrication. The device was assembled using two $4.75 \mathrm{~mm}$ by $6.2 \mathrm{~mm}$ microfluidic chambers separated by a semipermeable polycarbonate membrane filters (3.0 $\mu \mathrm{M}, 13 \mathrm{~mm}$; SterliteCH, M-170098, Kent, Washington, USA). The complete device was fabricated by soft lithography in polydimethylsiloxane (PDMS, Sylgard 184, Dow Corning, Midland, Michigan, USA) as previously described in Gnecco et al. ${ }^{7,16,17}$

Assembly of the 2-chambers platform. The top PDMS layer and the membrane were oxygen-plasma treated (600 mTorr, 100 $\mathrm{W}, 45 \mathrm{~s}$ ) and bonded together. The membrane was then placed over the PDMS with forceps. The second layer was then bonded with the same method on the membrane orthogonally to the top PDMS layer. Oxygen-plasma treatment renders the exposed surfaces hydrophilic. Hence, the assembled devices were immediately filled with sterile DI $\mathrm{H}_{2} \mathrm{O}$ and stored at $4^{\circ} \mathrm{C}$ until used. For static experiments, $500 \mu \mathrm{L}$ cloning cylinders (Fisher Scientific, Pittsburgh, Pennsylvania) were bonded with liquid PDMS to the inlet/outlet regions of each channel to form small reservoirs for the cell media (Figure 1). Our primary 


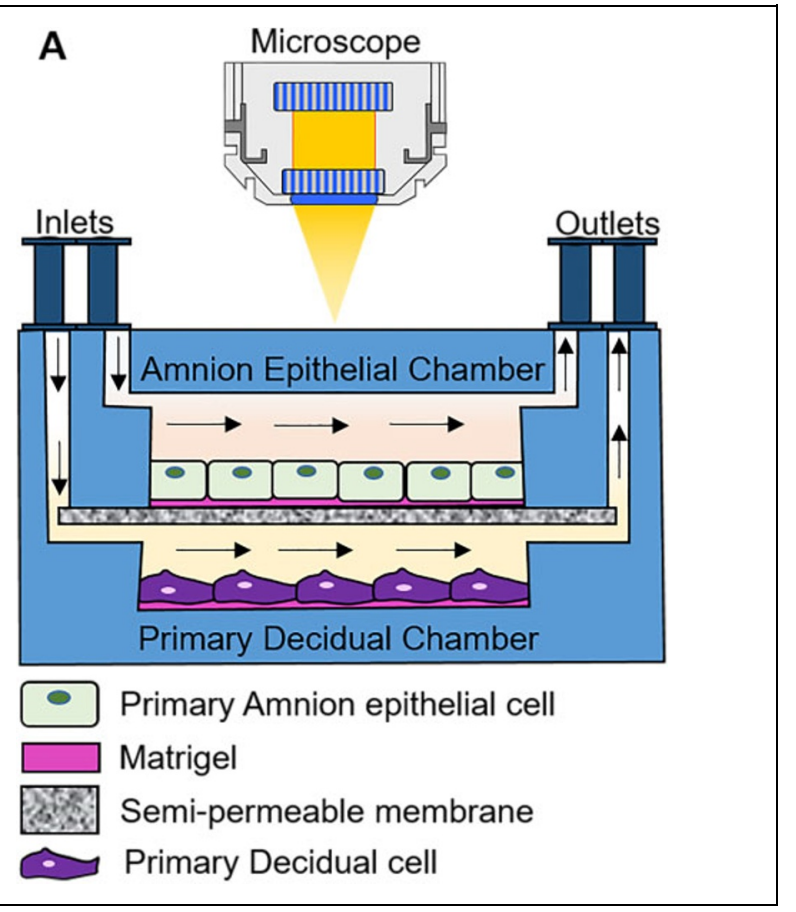

Figure I. Fetal membrane organ-on-chip schematic. Schematic of the 2-chambered fetal membrane organ-on-chip coated in matrigel. Amnion epithelial cells (AEC) are placed on top of a polycarbonate semipermeable membrane, while decidual cells are placed in a lower chamber.

objective of this descriptive study is to show the usefulness of FM-OO-C model compared to the transwell system and to project its future advantages.

\section{Clinical Samples and Cell Culture}

Amnion epithelial cell culture. Human primary AECs were isolated from amnion membrane obtained from fetal membranes from term, not in labor, cesareans. Approximately $10 \mathrm{~g}$ of amnion membrane peeled from the chorion layer were dispersed by successive treatments with $0.125 \%$ collagenase and $1.2 \%$ trypsin. All cell culture reagents were purchased from SigmaAldrich (St Louis, Missouri). Details of AEC isolation protocols can be found in our previous reports. ${ }^{3,4}$ Briefly, the dispersed cells were plated in a 1:1 mixture of Ham's F12/Dulbecco Modified Eagle Medium (DMEM), supplemented with $10 \%$ heatinactivated fetal bovine serum (FBS), $10 \mathrm{ng} / \mathrm{mL}$ epidermal growth factor (EGF), $2 \mathrm{mM}$ L-glutamine, $100 \mathrm{U} / \mathrm{mL}$ penicillin $\mathrm{G}$ and $100 \mathrm{mg} / \mathrm{mL}$ streptomycin at a density of 3 to 5 million cells per T75 and incubated at $37^{\circ} \mathrm{C}$ with $5 \% \mathrm{CO}_{2}$ until $80 \%$ to $90 \%$ confluence was achieved. The metastate (coexpression of epithelial and mesenchymal markers $)^{25}$ nature of the primary AECs in cultures was verified by immunocytochemistry as reported in our prior reports, and all cultures contained coexpression of cytokeratin-18 and vimentin positive cells.

Decidual cell culture. Primary decidual cells were isolated and scraped off the chorion from term not in labor fetal membranes, that is, decidualized endometrial cells of feto-maternal interface. Separation of the decidua involved blunt dissection with forceps and a scalpel. The decidual and chorion layers were minced by cross-cutting with scalpel blades. Tissues were processed in a digestion buffer containing $0.125 \%$ trypsin (Cat\# 85450 c, Sigma-Aldrich), $0.2 \%$ collagenase (Cat\# C0130, Sigma-Aldrich), and 0.02\% DNase I (Cat\# DN25, SigmaAldrich) and incubated at $37^{\circ} \mathrm{C}$ for 60 to 90 minutes. Samples were subsequently neutralized with complete media (1:1 mixture of Ham's F12/DMEM, supplemented with 5\% heatinactivated FBS, $10 \mathrm{ng} / \mathrm{mL}$ EGF, $100 \mathrm{U} / \mathrm{mL}$ penicillin $\mathrm{G}$, and $100 \mathrm{mg} / \mathrm{mL}$ streptomycin; Cat\# 30-001-CI, Corning, New York, USA). After filtration, the cell solution was centrifuged at $3000 \mathrm{rpm}$ for 10 minutes. A cell separation gradient was prepared using an Optiprep column (Axis-Shield, Dundee, United Kingdom), with steps ranging from $4 \%$ to $40 \%$ of 4 $\mathrm{mL}$ each $(4 \%, 6 \%, 8 \%, 9 \%, 10 \%, 20 \%, 30 \%$, and $40 \%)$. Digested decidual cells were added to the top of the gradient and centrifuged $(3000 \times g)$ at room temperature for 35 minutes. Cell densities of 1.027 to $1.038 \mathrm{~g} / \mathrm{mL}$ represented cell found within the decidual layer. Harvested cells were washed with DMEM, centrifuged, and resuspended in DMEM, and cell viability was tested using the trypan blue exclusion method. The resuspended cells were subsequently seeded at a density of 3 to 5 million cells per T75 and incubated at $37^{\circ} \mathrm{C}$ with $5 \% \mathrm{CO}_{2}$ until $80 \%$ to $90 \%$ confluence was achieved.

\section{Cell Culture Seeding}

Before using the FM-OO-C or transwells $(3.0 \mu \mathrm{m}$ pore size), devices were washed 3 times with $1 \times$ phosphate-buffered saline (PBS), coated with Matrigel (Corning Matrigel Basement Membrane Matrix, ${ }^{*}$ LDEV-free; 1:50 in PBS), and incubated at $37^{\circ} \mathrm{C}$ with $5 \% \mathrm{CO}_{2}$ for 30 minutes. Next, devices were washed 3 times with complete DMEM/F12 media before cell seeding. Primary cells were then trypsinized and 25000 AECs in the top chamber and 15000 Decidua in the bottom chamber of the FM-OO-C; while 50000 AEC (passage 1) and 30000 Decidua (passage 6 and 8) were plated into the transwell (Figures $1,2 \mathrm{~A}-\mathrm{C}$, and $3 \mathrm{~A}-\mathrm{C}$ ). Devices were incubated at $37^{\circ} \mathrm{C}$ with $5 \% \mathrm{CO}_{2}$ for 24 hours before treatment.

\section{Cell Culture Treatments}

To test the effect of OS and environmental toxins on signal propagation and senescence at the fetal-maternal interface, we treated each OOC with one of the following: (1) DMEM/F12 media (control), (2) OS inducer CSE 1:50, ${ }^{3}$ or (3) environmental toxin 2,3,7,8-tetrachlorodibeno-p-dioxin $(10 \mathrm{nM})$. Once cells reached $70 \%$ to $80 \%$ confluence, each OOC was rinsed with sterile $1 \times \mathrm{PBS}$, treated, and incubated at $37^{\circ} \mathrm{C}, 5 \% \mathrm{CO}_{2}$, and $95 \%$ air humidity for 48 hours. After 48 hours, bright field microscopy (Nikon Eclipse TS100 microscope $(10 \times$; Nikon, Melville, New York) was performed to determine cell morphology and staining for each treatment. 


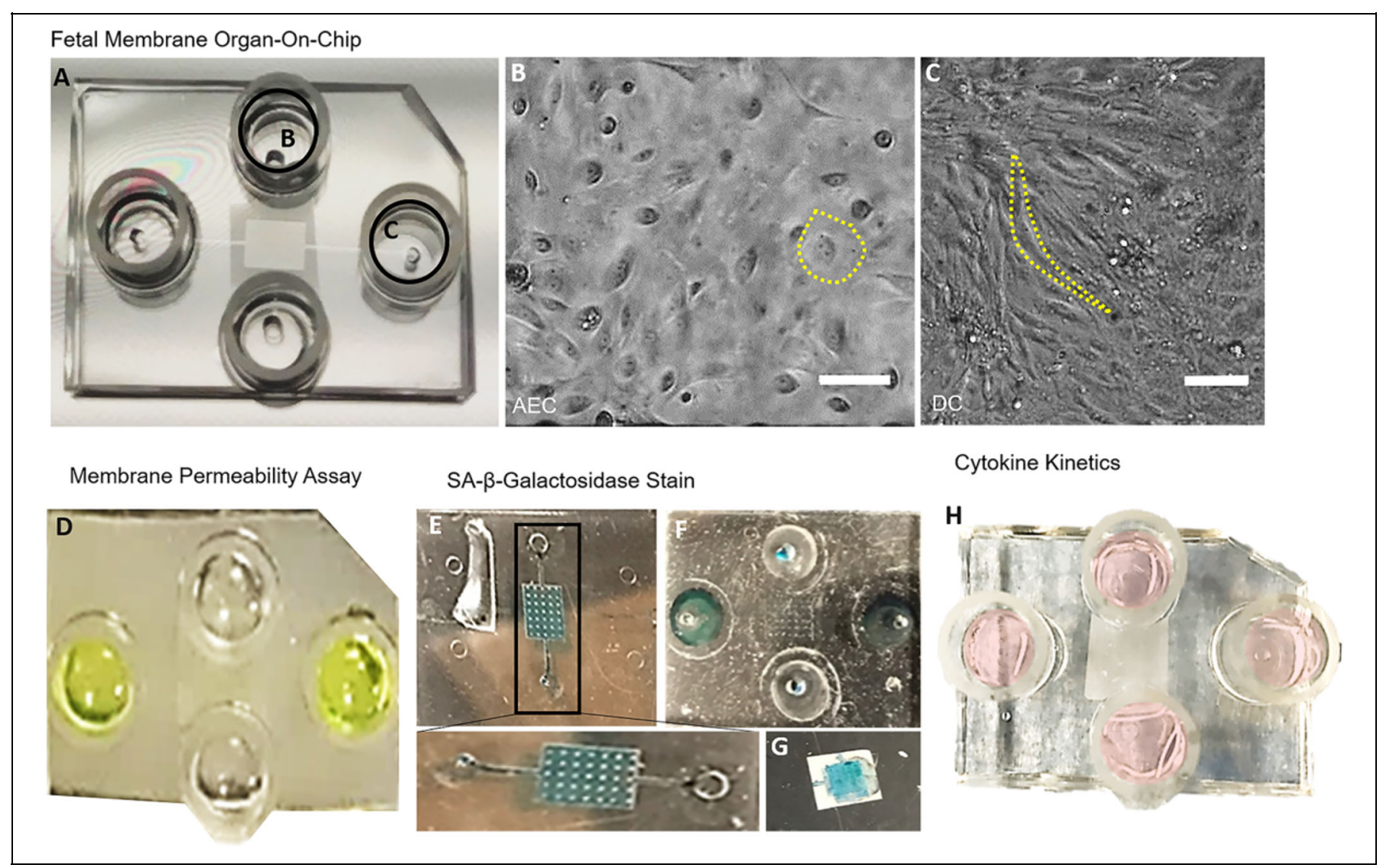

Figure 2. Fetal membrane organ-on-chip experimental layout and outcomes. A, Images of the FM-OO-C layout and experimental design. Fetal membrane-organ-on-chip is a 2-chamber device divided by a polycarbonate semipermeable membrane. B, Bright field microscopy image of primary human AECs in the top chamber and (C) primary decidual cells (DC) in the bottom chamber. Yellow outline documents morphology. Images were captured at $10 \times$ and contain $20-\mu \mathrm{m}$ scale bars. D, FITC stain (yellow) is seen in the 2 horizontal columns feeding into the top chamber AECs. Media were collected from the bottom vertical columns to measure membrane permeability. E, Bottom chamber showing representative SA- $\beta-G$ al stained decidual cells and $(F)$ top chamber of the FM-OO-C along with the (G) the semipermeable membrane containing blue staining representing SA- $\beta-$ Gal + AECs. H, Image of FM-OO-C containing media from both amnion and decidual cells which can be used to measure cytokine kinetics. AEC indicates amnion epithelial cells; FITC, fluorescein isothiocyanate; FM-OO-C, fetal membraneorgan-on-chip; SA- $\beta$-Gal, senescence-associated $\beta$-galactosidase.

\section{Fluorescein Isothiocyanate Membrane Perfusion Assay}

To determine membrane permeability and media propagation between both chambers of the FM-OO-C and transwell devices, we designed a set of perfusion assay experiments (Figures 2D and 3D). Fluorescein isothiocyanate (FITC) was added to the top insert and chamber of the devices while PBS was added to the bottom. Collected effluent from the bottom compartment was collected for up to 2 hours to determine membrane integrity. A fluorescence microplate reader (GloMax Multimode Readers, Promega, Madison, Wisconsin, USA) at $470 \mathrm{~nm}$ excitation and 520 to $550 \mathrm{~nm}$ emission range was used to measure the intensity of FITC dye that perfused through the membrane layer and into the bottom chamber.

\section{Senescence-Associated $\beta$-Galactosidase Assay}

Expression of the senescence-associated $\beta$-galactosidase (SA$\beta$-Gal) biomarker is independent of DNA synthesis and distinguishes senescent cells from normal cells. ${ }^{4,24}$ Senescent cells were identified using a histochemical staining kit (ab65351), with blue cells visualized by light microscopy at 48 hours after treatment (Figures 2E and 3E). An average of 4 regions were collected per condition using bright-field microscopy (Nikon Eclipse TS100 (10×; Melville, New York) and the number of cells with positive staining (blue colored cells) were analyzed. Image modifications (brightness, contrast, and smoothing) were applied to all image sets using Lightroom and Image J (National Institutes of Health, rsbweb.nih.gov/ij) and a grading scale was used to measure the staining intensity of 5 image fields per experiment (Figure 4B).

\section{Statistics}

For this descriptive manuscript, a total of 4 cell preparations ( 2 AECs and 2 decidual cell preparations) were used to validate this method. Thus, fold change and trends were reported instead of statistical significance. 


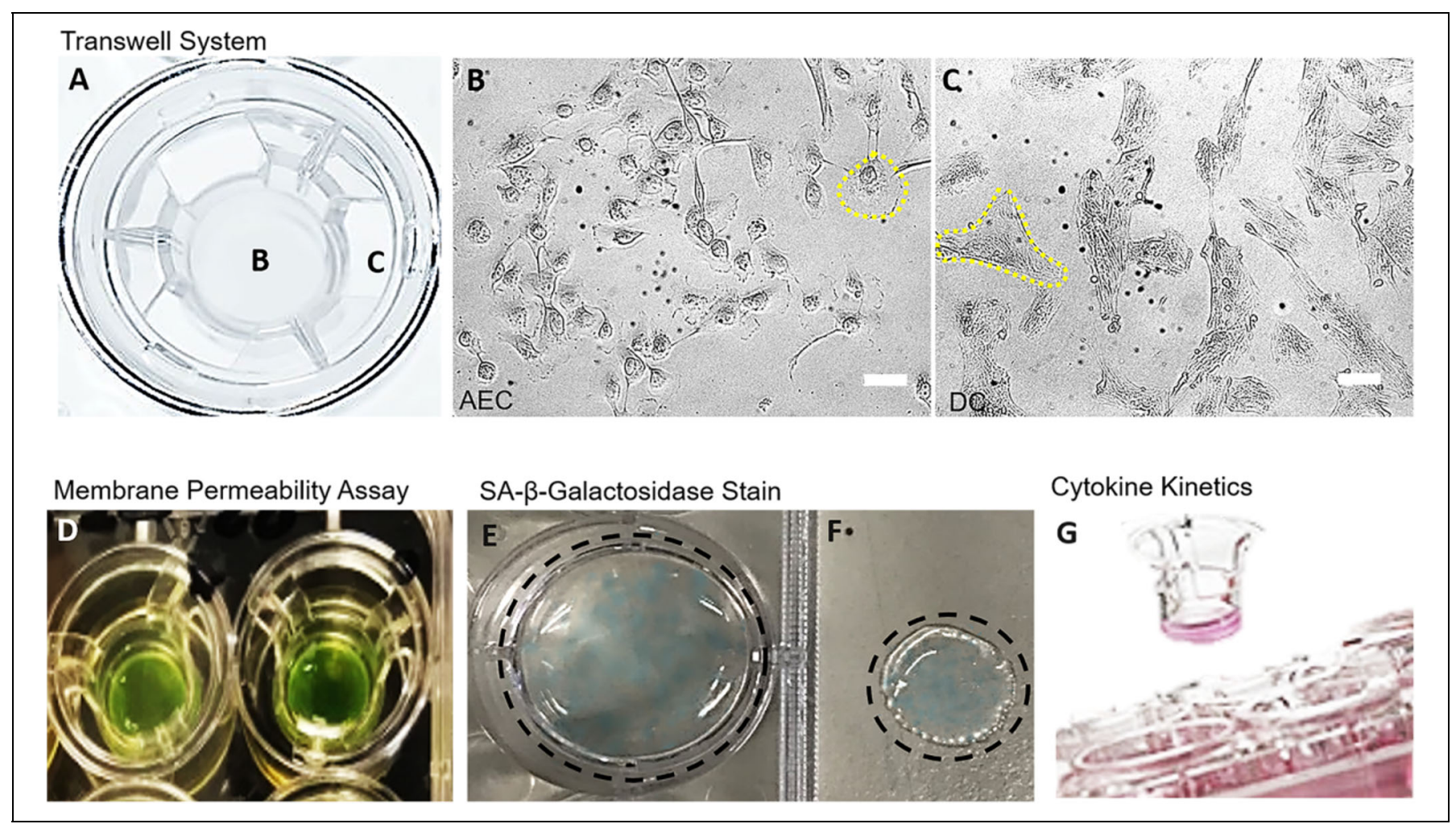

Figure 3. Transwell experimental layout and outcomes. A, Images of the transwell layout and experimental design. Transwell is a 2-chamber device divided by a polycarbonate semipermeable membrane. B, Bright field microscopy image of primary human AECs in the top chamber and (C) primary decidual cells (DC) in the bottom chamber of the transwell devices. Yellow outline documents morphology. Images were captured at $10 \times$ and contain $20-\mu \mathrm{m}$ scale bars. D, FITC stain is seen in the 2 columns (top chamber) feeding into the top chamber AECs. Media were collected from the bottom columns (bottom chamber) to measure membrane permeability. E, The lower chamber of the transwell showing representative SA- $\beta$-Gal stained decidua (blue). F, The semipermeable membrane containing blue staining representing SA- $\beta-G a l+$ AECs are circled in black. G, Image of transwell devices containing media from both amnion and decidual cells which can be used to measure cytokine kinetics. AEC indicates amnion epithelial cells; FITC, fluorescein isothiocyanate; SA- $\beta$-Gal, senescence-associated $\beta$-galactosidase.

\section{Results}

\section{Development of FM-OO-C}

The FM-OO-C microfluidic coculture platform was composed of 2 orthogonal stacked chambers, containing equal surface area $\left(29.45 \mathrm{~mm}^{2}\right)$ that contained primary AECs (fetal) on top and decidual cells (maternal) on the bottom (Figures 1 and $2 \mathrm{~A}$ C). The 2 chambers are divided by a semipermeable membrane similar to extracellular matrix component of fetal membranes (Figure 2E, yellow circle). Additionally, this membrane allowed effluent signal propagation and cell migration between compartments permitting for in vitro analysis of complex biological processes (Figure 2D-H). The same cell preparations in traditional transwell cultures were used for comparison purposes (Figure 3).

\section{Cellular Interactions Are Better Determined Using FM-OO-C Than Transwell Cultures}

To compare the FM-OO-C to a more conventional transwell, we treated AEC and decidual cells separately with 2 environmental toxicants (cigarette smoke and dioxin) and examined the changes on AECs and its impact on decidual cells and vice versa. As shown in Figure 4, dioxin exposure differentially induced membrane permeability at the fetal-maternal interface, compared to the OS-inducer CSE in the FM-OO-C. Compared to transwell cultures, the 2-chamber FM-OO-C model promoted better cellular interactions and allowed sensitive measurement of membrane permeability irrespective of treatment side (AEC vs decidual cells; Figure 4A). Cigarette smoke extract treatment of AEC produced higher membrane permeabilization (1.6-fold) than treatment on the decidual side (1.2-fold); whereas dioxin treatment on the decidual cell side produced higher permeabilization (1.1-fold) than treatment of the same on AEC side ( 0.7 -fold; Figure $4 \mathrm{~A})$. In contrast, this effect was minimal in corresponding transwell treatments.

\section{Fetal Membrane-Organ-On-Chip Promotes Biochemical Exchange Between Fetal and Maternal Chambers Leading to Senescence}

To identify the ability of OS (CSE treatment) and environmental toxins (dioxin treatment) to induce and propagate signals of cellular senescence between the 2 chambers of the FM-OO-C, 
A

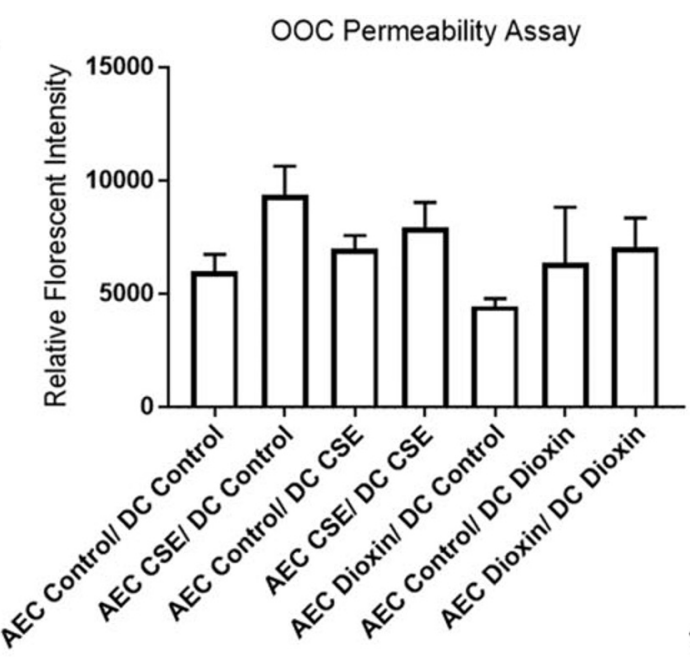

Transwell Permeability Assay

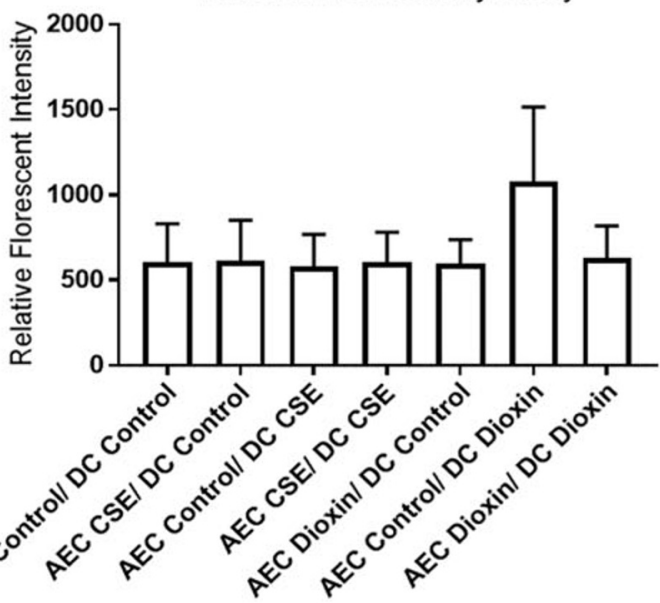

B

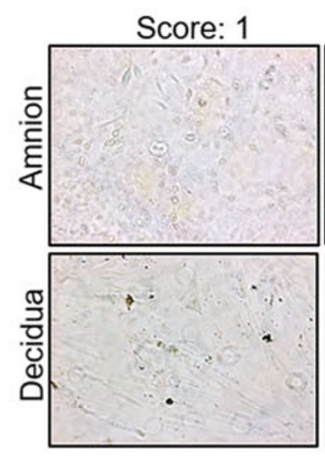

Score: 2

Score: 3

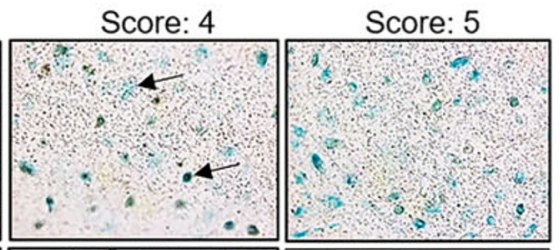

C
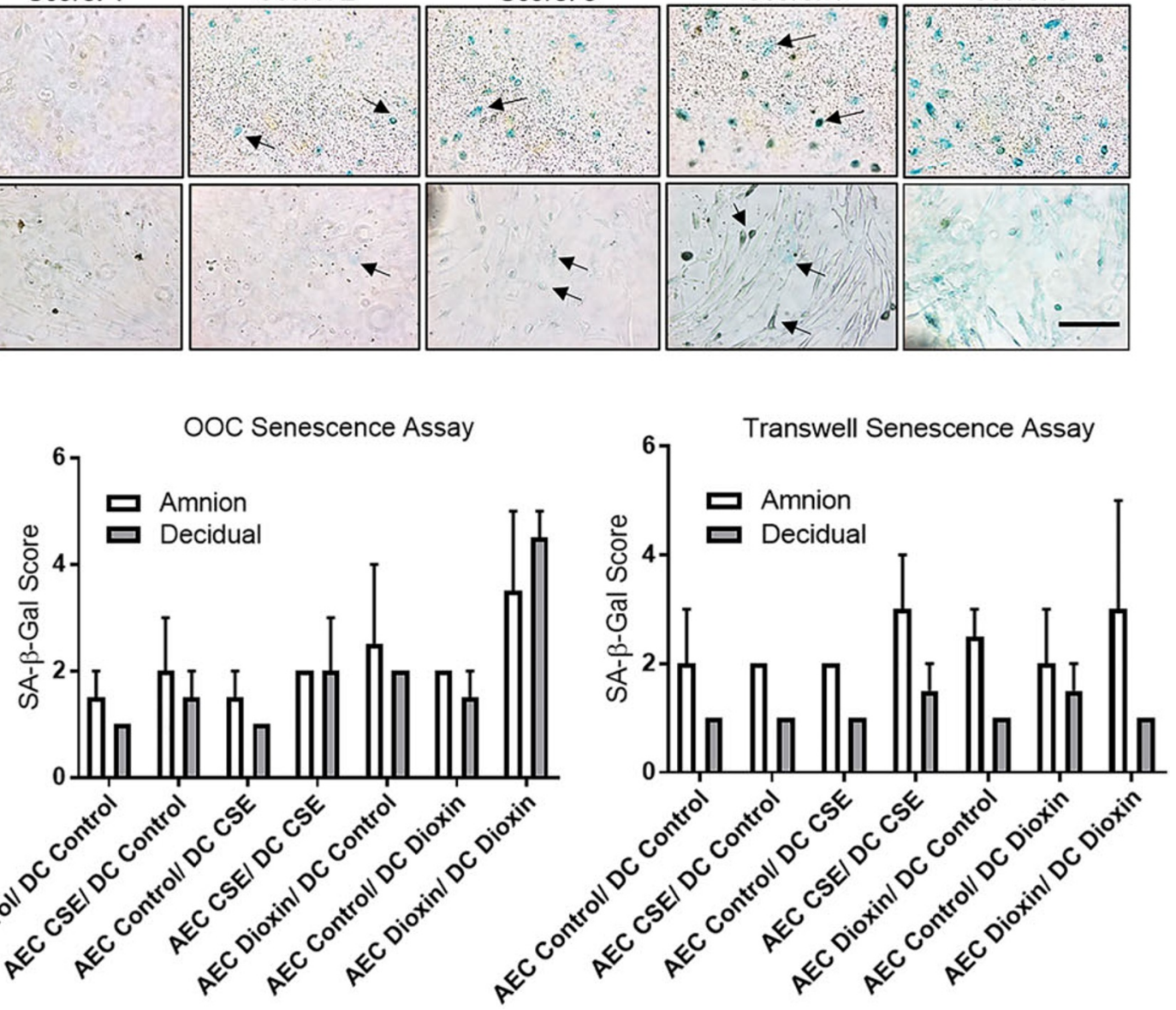

Figure 4. Cigarette smoke extract (CSE) and dioxin differentially induce membrane permeability and senescence in fetal and maternal cells. A, Fetal membrane-organ-on-chip (FM-OO-C) and transwell devices were seeded with AEC and decidual cells (AEC/decidual cells), treated with either CSE or dioxin media for 48 hours. Cells were also maintained in cell culture media as controls. Fluorescein isothiocyanate measured membrane permeability for up to 2 hours. Compared to transwell cultures, the 2-chamber FM-OO-C allowed for more sensitive measurement of membrane permeability regardless of treatment side. Membrane permeabilization was higher with AECs treated with CSE compared to similar treatment on the decidual side. Decidual cells treated with dioxin-induced higher membrane permeabilization compared to dioxin treatment on AEC side in OOC and transwell systems ( 2 fold). B, Scoring system to determine the amount of SA- $\beta$-Gal (blue; arrows) in AECs and decidual cell cultures. All images were taken at $20 \times$ and scale bar is set to 20 microns. $C$, Relative quantitation of SA- $\beta$-Gal stain from the FM-OO-C and transwell devices. Treatments are documented as (AEC/DC). AEC indicates amnion epithelial cells; FM-OO-C, fetal membraneorgan-on-chip; OOC, organ-on-chip; SA- $\beta$-Gal, senescence-associated $\beta$-galactosidase. 
we designed a scoring system for SA- $\beta$-Gal staining (blue) in AECs and decidual cells (Figure 4B). Compared to control cultures, CSE and dioxin treatments induced cellular senescence in AECs and decidual cells after 48 hours. Additionally, both CSE- and dioxin-treated sides in the FM-OO-C induced senescence and forced similar changes on the opposite chamber (ie, decidua treatment of CSE-induced AEC senescence 1.4fold higher than controls, while AEC treatment with dioxininduced decidual senescence 1.4-fold higher than controls), suggesting transfer of biochemical signals between layers that are capable of producing labor-associated changes (Figure 4C). Transwell cultures compared to the corresponding FM-OO-C treatments did not allow propagation of such signals as indicated by minimal effect on SA- $\beta$-Gal levels (Figure 4C).

\section{Discussion}

Here, we further developed an organ-on-chip (OOC) model of the human fetal membrane with a device containing human AECs and decidual cells, a model for the fetal-maternal interface component of the fetal membranes that can be used to test cell migrations and transitions, intercellular interactions, and signaling. Amnion epithelial cells and decidual cells were cocultured in a 2-chamber OOC separated by a semipermeable membrane representing the fetal membrane extracellular matrix. This device allowed us to test fetally derived signals' ability to induce extracellular matrix permeability and cellular changes like senescence. Here, we document that the FM-OO$\mathrm{C}$ model (1) maintains physical and fluidic isolation between fetal and maternal compartments (cells), (2) stimulates primary cells to cause biochemical changes, (3) provides better interactions between cell types, and (4) allows for better transfer of biochemicals between compartments compared to transwell cultures to impact changes by the recipient cells. Though more rigorous testing should be conducted before making a definitive statement that the FM-OO-C model is better than transwells, these results suggest OOC devices are ideal for addressing specific questions related to feto-maternal signaling during pregnancy.

The controlled environment of the FM-OO-C allows improved signal propagation between cells/chambers by minimizing variability while highlighting subtle changes like cell's ability to induce membrane permeability and transfer biochemicals between treatments compared to transwell systems. ${ }^{7,16,17}$ On the contrary, transwell systems by nature enhance their nascent signal/noise ratio with different surface areas, cell quantities, and media volumes between the 2 chambers. Specifically, negative results in transwell experiments, in part, could be due to the large difference in effluent volume between the top and bottom chambers of the transwell (Top: $600 \mu \mathrm{L}$; Bottom: $1500 \mu \mathrm{L}$ ) which is addressed in most organ-on-chip models (FM-OO-C; top: $800 \mu \mathrm{L}$; bottom: $800 \mu \mathrm{L}$ ). This difference could be one of the reasons fetal and maternal biochemical signals failed to propagate and induce senescence in the transwell system compared to the FM-OO-C. Additionally, stimulant-dependent changes were not detected in transwell membrane perfusion studies. Dilution of the transwell FITC signal (10-fold lower), which crossed to the lower chamber during the membrane perfusion assay, could address these findings. Due to FM-OO-C's ability to amplify small changes in effluent signals, future experiments will be conducted to utilize dynamic flow of media in this system to replicate shear stress at the cellular level and to detect the kinetics of various signal generation.

Although FM-OO-C has many advantages to single cell cultures, the model presented here has limitations. Besides the dynamic flow system mentioned above, some other areas to be addressed in future designs include (1) composition of the semipermeable membrane to better mimic extracellular matrix in utero, (2) organization of the device to allow for better imaging of chambers and migratory cells, (3) the addition of other collagen types and cell layers to complete the fetalmaternal interface. This model did not include amnion mesenchymal cells, chorionic trophoblasts, or immune cells that also play a major role in signaling between AEC and decidual cells, ${ }^{16,18}$ and (4) adaptations in cell loading to produce an even and consistent cell confluence.

In summary, we utilized an OOC model to recreate the fetal-maternal compartment of the fetal membranes (FMOO-C), which can be used to better understand cellular interactions and paracrine cross-talk between maternal and fetal cells during pregnancy and parturition. Organ-on-chips representing a variety of physiological states and organ systems have become a critical step in the drug discovery pipeline and are the future of bench to bedside research. Continual progress to the FM-OO-C model is expected to recreate the entire fetal membrane-on-chip, thus allowing for a full model of pregnancy-on-chip for studying cellular interactions during pregnancy and parturition, screening of drugs, and to advance research activities to reduce the risk of pregnancy-associated complications.

\section{Authors' Note}

The data sets generated during and/or analyzed during the current study are available from the corresponding author on reasonable request. Its contents are solely the responsibility of the grantees and do not necessarily represent the official views of the US NIH or EPA. Further, US EPA does not endorse the purchase of any commercial products or services mentioned in the publication.

\section{Acknowledgments}

Lauren Richardson is an appointed Predoctoral Trainee in the Environmental Toxicology (ETox) Training Program (T32ES007254), supported by the National Institute of Environmental Health Sciences (NIEHS) of the National Institutes of Health (NIH) of the United States, and administered through the University of Texas Medical Branch in Galveston, Texas.

\section{Declaration of Conflicting Interests}

The author(s) declared no potential conflicts of interest with respect to the research, authorship, and/or publication of this article. 


\section{Funding}

The author(s) disclosed receipt of the following financial support for the research, authorship, and/or publication of this article: Grant AI134036 from the National Institutes of Health (NIH), Research Grant from the March of Dimes, the Department of Veteran Affairs (BX002853), and by US Environmental Protection Agency (EPA) Grant \#83573601. This study was also supported by the NICHD (grant number 1R01HD084532-01A1) to R. Menon.

\section{ORCID iD}

David M. Aronoff (D) https://orcid.org/0000-0003-4587-6121

\section{References}

1. Behnia F, Taylor BD, Woodson M, et al. Chorioamniotic membrane senescence: a signal for parturition? Am J Obstet Gynecol. 2015;213(3):359.e1-416.e1.

2. Bredeson S, Papaconstantinou J, Deford JH, et al. HMGB1 promotes a p38MAPK associated non-infectious inflammatory response pathway in human fetal membranes. PLoS One. 2014; 9(12):e113799.

3. Dixon CL, Richardson L, Sheller-Miller S, Saade G, Menon R. A distinct mechanism of senescence activation in amnion epithelial cells by infection, inflammation, and oxidative stress. $A m J$ Reprod Immunol. 2017;79(3).

4. Menon R, Boldogh I, Urrabaz-Garza R, et al. Senescence of primary amniotic cells via oxidative DNA damage. PLoS One. 2013; 8(12):e83416.

5. Menon R, Boldogh I, Hawkins HK, et al. Histological evidence of oxidative stress and premature senescence in preterm premature rupture of the human fetal membranes recapitulated in vitro. Am J Pathol. 2014;184(6):1740-1751.

6. Menon R, Bonney EA, Condon J, Mesiano S, Taylor RN. Novel concepts on pregnancy clocks and alarms: redundancy and synergy in human parturition. Hum Reprod Update. 2016;22(5): 535-560.

7. Gnecco J, Osteen K. Compartmentalized culture of perivascular stroma and endothelial cells. Biomedical Engineering Society. 2017;45(7):1758-1769.

8. Duval K, Grover H, Han LH, et al. Modeling physiological events in 2D vs. 3D cell culture. Physiology (Bethesda). 2017;32(4): 266-277.

9. Katt ME, Placone AL, Wong AD, Xu ZS, Searson PC. In vitro tumor models: advantages, disadvantages, variables, and selecting the right platform. Front Bioeng Biotechnol. 2016;4:12.

10. Sances S, Ho R, Vatine G, et al. Svendsen, human iPSC-derived endothelial cells and microengineered organ-chip enhance neuronal development. Stem Cell Reports. 2018;10(4):1222-1236.
11. van der Helm MW, van der Meer AD, Eijkel JC, van den Berg A, Segerink LI. Microfluidic organ-on-chip technology for bloodbrain barrier research. Tissue Barriers. 2016;4(1):e1142493.

12. Huh DD. A human breathing lung-on-a-chip. Ann Am Thorac Soc. 2015;12(suppl 1):S42-S44.

13. Konar D, Devarasetty M, Yildiz DV, Atala A, Murphy SV. Lungon-a-chip technologies for disease modeling and drug development. Biomed Eng Comput Biol. 2016;7(suppl 1):17-27.

14. Zhang YS, Aleman J, Arneri A, et al. From cardiac tissue engineering to heart-on-a-chip: beating challenges. Biomed Mater. 2015;10(3):034006.

15. Materne EM, Maschmeyer I, Lorenz AK, et al. The multi-organ chip - a microfluidic platform for long-term multi-tissue coculture. J Vis Exp. 2015;(98):e52526.

16. Gnecco JS, Anders AP, Cliffel D, et al. Instrumenting a fetal membrane on a chip as emerging technology for preterm birth research. Curr Pharm Des. 2017;23(40):6115-6124.

17. Bruner-Tran KL, Gnecco J, Ding T, Glore DR, Pensabene V, Osteen KG. Exposure to the environmental endocrine disruptor TCDD and human reproductive dysfunction: translating lessons from murine models. Reprod Toxicol. 2017;68:59-71.

18. Anders AP, Gaddy JA, Doster RS, Aronoff DM. Current concepts in maternal-fetal immunology: recognition and response to microbial pathogens by decidual stromal cells. Am J Reprod Immunol. 2017;77(3).

19. Richardson L, Vargas G, Brown T, et al. Redefining 3Dimensional placental membrane microarchitecture using multiphoton microscopy and optical clearing. 2017;53:66-75.

20. Rogers L, Anjali A, Doster R, et al. Decidual stromal cell-derived PGE2 regulates macrophage responses to microbial threat. $\mathrm{Am} \mathrm{J}$ Reprod Immunol. 2018;80(4):e13032.

21. Menon R, Fortunato SJ, Yu J, et al. Cigarette smoke induces oxidative stress and apoptosis in normal term fetal membranes. Placenta. 2011;32(4):317-322.

22. Sheller-Miller S, Urrabaz-Garza R, Saade G, Menon R. Damageassociated molecular pattern markers HMGB1 and cell-free fetal telomere fragments in oxidative-stressed amnion epithelial cellderived exosomes. J Reprod Immunol. 2017;123:3-11.

23. Abe Y, Sinozaki H, Takagi T, et al. Identification of 2,3,7,8tetrachlorodibenzo-p-dioxin (TCDD)-inducible genes in human amniotic epithelial cells. Reprod Biol Endocrinol. 2006;4:27.

24. Jin J, Richardson L, Sheller-Miller S, Zhong N, Menon R. Oxidative stress induces p38MAPK-dependent senescence in the feto-maternal interface cells. Placenta. 2018;67:15-23.

25. Richardson L, Menon R. Proliferative, migratory, and transition properties reveal metastate of human amnion cells. Am J Pathol. 2018;188(9):2004-2015. 